Nota de investigación

\title{
Razas fisiológicas de Puccinia triticina E. identificadas en el norte de Sinaloa y resistencia de germoplasma
}

\author{
María Florencia Rodríguez García ${ }^{1}$ \\ Elizabeth García León ${ }^{2 \S}$ \\ Julio Huerta Espino ${ }^{1}$ \\ Héctor Eduardo Villaseñor Mir ${ }^{1}$ \\ Genny Llaven Valencia² \\ Miguel González González ${ }^{1}$
}

${ }^{1}$ Campo Experimental Valle de México-INIFAP. Carretera Los Reyes-Texcoco km 13.5, Coatlinchán, Texcoco, Estado de México. (rodriguez.maria@inifap.gob.mx; j.huerta@cgiar.org; (hevimir3@yahoo.com.mx; gonzalez.miguel@inifap.gob.mx). ${ }^{2}$ Campo Experimental Valle del FuerteINIFAP. Carretera México-Nogales km 1609, Juan José Ríos, Guasave, Sinaloa. CP. 81110. (llaven.genny@inifap.gob.mx).

${ }^{\S}$ Autora para correspondencia: garcia.elizabeth@inifap.gob.mx.

\section{Resumen}

La roya de la hoja causada por Puccinia triticina E. es la enfermedad más importante en el norte y noroeste de México. En el norte de Sinaloa está ampliamente distribuida en las zonas de producción de trigo del Valle del Fuerte y Valle del Carrizo; sin embargo, se desconoce que razas están incidiendo en dicha región. El objetivo fue identificar las razas fisiológicas de roya de la hoja, que se presentan en las principales zonas productoras del norte de Sinaloa e identificar germoplasma con resistencia. Durante el ciclo de cultivo otoño-invierno 2018-2019, se colectaron 50 muestras de roya de la hoja de lotes experimentales y comerciales, dichas muestras se trasladaron al Lanarec. Con la técnica de monopostulares, se realizó la identificación de razas. Posteriormente con las razas más frecuentes se evaluaron bajo condiciones de invernadero y por su resistencia en plántula y planta adulta 35 genotipos de trigo harinero. Se identificaron siete razas fisiológicas de roya de la hoja (CBJ/QQ, MBT/SP, MCT/SP, MGJ/SP, MBJ/SP, MBJ/QQ, MFJ/SP), de las cuales la $\mathrm{CBJ} / \mathrm{QQ}$ se identificó con mayor frecuencia (29\%). De los 35 genotipos evaluados con las razas CBJ/QQ y MCT/SP, se determinó que CONATRIGO F2015 y las líneas CHEWINK \#1/MUTUS//MUTUS*2/HARIL\#1 y MUTUS*2//ND643/2*WBLL1/3/BORL14 fueron resistentes en plántula y planta adulta a las dos razas, lo que indica que posee al menos un gen de resistencia efectivo a roya de la hoja. La resistencia genética es una de las alternativas de manejo sustentable más viable para el control de las royas del trigo en México.

Palabras clave: genotipos, resistencia, virulencia.

Recibido: noviembre de 2020

Aceptado: diciembre de 2020 
A nivel mundial el trigo harinero (Triticum aestivum L.) es el cereal de mayor importancia después del maíz, su producción es principalmente para consumo humano (FAO, 2020). En México las principales regiones productoras de trigo se ubican en el Noroeste (Sinaloa, Sonora y Baja California), donde se produce $64.2 \%$ del total nacional, dicha producción se realiza bajo condiciones de riego durante el ciclo otoño-invierno (O-I) (SIAP, 2020).

En Sinaloa la zona triguera comprende los municipios de Guasave, Sinaloa de Leyva, Ahome, Angostura y El Fuerte. El municipio de Ahome concentra más de $80 \%$ de la producción total del estado, destacando el Valle del Carrizo como la principal área triguera con 33350 ha sembradas en el ciclo O-I 2018-2019 (CESAVESIN, 2019).

La producción de trigo es afectada por factores bióticos y abióticos. Entre los factores bióticos, las enfermedades como las royas, ocasionadas por diferentes especies del género Puccinia son las económicamente más importantes del cultivo de trigo en México. La roya de la hoja causada por Puccinia triticina E. es la más importante en el norte y noroeste del país, dicha enfermedad es la más distribuida y se presenta en la mayoría de las zonas de riego donde se siembra trigo (HuertaEspino et al., 2011).

Singh et al. (2016) indican que las royas históricamente han causado grandes pérdidas en el cultivo de trigo y siguen siendo económicamente importantes a pesar del uso generalizado del control genético y químico. Por otra parte, Rodríguez et al. (2010) mencionan que las royas constantemente amenazan la producción de este cereal en el mundo, dado que pueden vencer la resistencia especifica de variedades nuevas por la evolución hacia nuevos biotipos del patógeno o razas fisiológicas con nuevos genes de virulencia; además se reproducen rápidamente y pueden moverse a distancias muy grandes. La roya de la hoja ha causado pérdidas en el rendimiento que van desde 10 a 50\% (Huerta-Espino et al., 2011).

En roya de la hoja existe una marcada diferencia entre las poblaciones del patógeno que atacan a trigos harineros (Triticum aestivum L.) (Singh, 1991), respecto a las que atacan a los trigos duros (Triticum durum L.) (Huerta-Espino y Roelfs 1989). En México Huerta-Espino y Singh (2000) indicaron que se han identificado más de 50 razas fisiológicas de roya de la hoja en trigo harinero, un cambio drástico de la población de este patógeno ocurrió en 1994 en el Sur de Sonora donde prevalecía la raza MCJ/SP (que eventualmente se hizo presente en los Valles Altos) pero en 1996, en Oaxaca se detectó por primera vez la raza M(B)J/SP, variante de la raza MCJ/SP. Por otra parte, Villaseñor et al. (2003) en el año 2000 en los Valles Altos de México identificó 13 razas fisiológicas que afectan a trigo harinero siendo MCJ/SP la más frecuente seguida de MBD/QM.

El control genético en royas está basado en utilizar variedades con resistencia a las diversas razas fisiológicas. Por esta razón, se busca generar variedades que posean resistencia durable (poligénica, multigénica, horizontal o cuantitativa) basada en genes de patogenia lenta slow rusting (Singh et al., 2011). Hasta la fecha se han catalogado 77 genes de resistencia para roya de la hoja (McIntosh et al. 2017). De los cuales, Lr12, Lr13, Lr22a, Lr22 b, Lr34, Lr35, Lr37, Lr46, Lr48, Lr49, Lr67, Lr68, $L r 74, L r 75$ confieren resistencia en planta adulta (RPA) (McIntosh et al., 2017).

En el norte de Sinaloa la roya de la hoja está ampliamente distribuida en las zonas de producción de trigo del Valle del Fuerte y Valle del Carrizo; sin embargo, se desconoce que razas fisiológicas están incidiendo en dicha región. El objetivo de esta investigación fue identificar las razas 
fisiológicas de roya de la hoja, que se presentaron en las principales zonas productoras del norte de Sinaloa durante el ciclo otoño-invierno 2018-2019 e identificar germoplasma con resistencia a las razas más comunes y con un mayor espectro de virulencia.

Durante el ciclo otoño-invierno 2018-2019 se colectaron en diferentes predios del Valle del Carrizo y Valle del Fuerte pertenecientes al norte de Sinaloa, 50 muestras de hojas con signos de roya de la hoja, anotando los datos de colecta. Las muestras se trasladaron al laboratorio nacional de royas y otras enfermedades de cereales (LANAREC) del INIFAP-CEVAMEX, ubicado en Texcoco, Estado de México.

\section{Identificación de razas fisiológicas}

Mediante la técnica de monopostulares y siguiendo la metodología descrita por Huerta-Espino et al. (2020) se obtuvieron 150 aislamientos. Para la identificación de razas fisiológicas se utilizó un conjunto de 20 líneas diferenciales, arregladas en cinco juegos de cuatro diferenciales cada una. Además de las 20 diferenciales, se sembraron otros 31 genotipos de trigo que permiten corroborar la virulencia y avirulencia del hongo en estos genes, así como la diferenciación de razas de trigos harineros vs trigos cristalinos.

A los 10 días después de la inoculación se registró la reacción de los genotipos utilizando la escala del 0-4 descrita por Roelfs et al. (1992). La nomenclatura para la designación de razas se basó en la propuesta por Long y Kolmer (1989), modificada por Singh (1991) y descrita para México por Huerta-Espino et al. (2020).

\section{Evaluación de genotipos en plántula y planta adulta}

En el LANAREC, bajo condiciones de invernadero ( $\left.\mathrm{T}_{\operatorname{máx}} 24{ }^{\circ} \mathrm{C}-\mathrm{T}_{\min } 18{ }^{\circ} \mathrm{C}\right)$ se evaluó la resistencia en plántula y planta adulta de ocho variedades testigo y 27 líneas avanzadas de trigo harinero, provenientes del programa de mejoramiento genético de trigo de temporal del INIFAPCEVAMEX. Para la evaluación en plántula se sembraron los 35 genotipos en charolas de plástico, se marcaron pequeños orificios y se colocaron de 8 a 9 semillas por genotipo, posteriormente a los 11 días después de la siembra las plántulas se inocularon con una suspensión de urediniosporas de las razas de roya de la hoja denominadas CBJ/QQ y MCT/SP, a una concentración de 1 x $10^{6}$ urediniosporas $\mathrm{ml}^{-1}$.

Las urediniosporas fueron suspendidas en aceite mineral (Soltrol ${ }^{\circledR} 170$; Chevron Phillips Chemical Company) y asperjadas con un atomizador conectado a un compresor. Las plántulas inoculadas se colocaron en una cámara bioclimática con temperaturas de $24{ }^{\circ} \mathrm{C}$ por $24 \mathrm{~h}$ y roció al $100 \%$. Posteriormente se trasladaron al invernadero y después de 10 días de inoculación se registró su reacción a la roya de la hoja utilizando la escala propuesta por Roelfs et al. (1992), donde lecturas del 0 al 2 se consideran resistentes y de 3 a 4 susceptibles.

Por otra parte, para la evaluación por su resistencia en planta adulta a RH los 35 genotipos fueron sembrados en macetas plásticas donde se colocaron tres semillas por maceta. A los 60 días después de la siembra, las plantas fueron inoculadas con una suspensión de las razas CBJ/QQ y MCT/SP, a una concentración de $1 \times 10^{6}$ urediniosporas $\mathrm{ml}^{-1}$. Las plantas inoculadas se colocaron en una cámara bioclimática con temperaturas de $24{ }^{\circ} \mathrm{C}$ por 24 h y roció al $100 \%$. 
Posteriormente se trasladaron al invernadero y después de 15 días de inoculación se registró la severidad de la enfermedad, utilizando la escala de Cobb modificada (Peterson et al. 1948) y la respuesta del hospedante a la infección se determinó de acuerdo con Roelfs et al. (1992). Donde: $\mathrm{R}=$ resistente, uredinios diminutos; $\mathrm{MR}=$ moderada resistencia, uredinios pequeños; $\mathrm{MS}=$ moderada susceptibilidad, uredinios pequeños de tamaño moderado; y $S=$ susceptible, uredinios grandes.

Se identificaron siete razas fisiológicas de roya de la hoja, que se presentaron en el ciclo otoñoinvierno/2018-2019 en el norte de Sinaloa, en el Cuadro 1, se presenta su fórmula de virulencia/avirulencia y en dicho cuadro se puede observar, que la raza más frecuente fue la CBJ/QQ con 29\% de frecuencia, dicha raza se identificó por primera vez en 1994 en los Valles Altos del centro México como lo menciona Huerta-Espino y Singh (1994) y comparte cierta similitud con la raza CBJ/QL y CBJ/QB reportadas por Singh (1991) identificada en el estado de Tlaxcala.

Cuadro 1. Razas fisiológicas de roya de la hoja identificadas en el norte de Sinaloa (ciclo O-I 2018-2019).

\begin{tabular}{cccc}
\hline No. & Razas & Frecuencia (\%) & Virulencia/avirulencia \\
\hline 1 & CBJ/QQ & 29 & Lr3,3bg,10,11,13,17,19/1,2a,2c,3ka,9,15,16,18,23,24,26,27+3,30 \\
2 & MBT/SP & 23 & Lr1,3,3ka,3bg,10,11,13,15,17,23,27+31,30/2a,2c,9,16,18,19,24,26 \\
3 & MCT/SP & 18 & Lr1,3,3ka,3bg,10,11,13,15,17,23,26,27+31,30,/2a,2c, $9,16,18,19,24$ \\
4 & MGJ/SP & 10 & Lr1,3,3bg,10,11,13,15,16,17,23,27+31/2a,2c,3ka,9,18,19,24,26,30 \\
5 & MBJ/SP & 10 & Lr1,3,3bg,10,11,13,15,17,23,27+31/2a,2c,3ka,9,16,18,19,24,26,30 \\
6 & MBJ/QQ & 5 & Lr1,3,3bg,10,11,13,17,19/2a,2c,3ka,9,15,16,18,23,24,26,27+31,30 \\
7 & MFJ/SP & 5 & Lr1,3,3bg,10,11,13,15,17,23,24,26,27+31/2a, 2c,3ka,9,16,18,19,30 \\
\hline
\end{tabular}

$\mathrm{Lr}=$ Leaf rust.

Por otra parte, la raza más frecuente fue la MBT/SP con 23\%, seguida de la MCT/SP (18\%), $\mathrm{MGJ} / \mathrm{SP}$ y MBJ/SP con $10 \%$ y con $5 \%$ de frecuencia las razas MBJ/QQ y MFJ/SP. Las razas con menos espectro de virulencia fue la CBJ/QQ y MBJ/QQ; sin embargo, esta última se identificó con menos frecuencia. Por otra parte, las razas más virulentas y con más frecuencia fueron MCT/SP y MBT/SP. De las siete razas identificadas se observó cierta similitud con otras razas reportadas principalmente en Estados Unidos de América, tal es el caso de la raza MBT/SP, dicha raza comparte similitud con la raza MBTSB identificada desde el 2005 y reportada por Kolmer et al. (2011); Kolmer y Hughes (2018).

La raza MFJ/SP se identificó similitud con la raza MFJSB reportada por Kolmer y Hughes (2018). En general la mayoría de las razas identificadas presentaron un espectro de virulencia amplio, lo que indica que a través del tiempo han evolucionado de una forma acelerada y han vencido la resistencia de la mayoría de los genes mayores disponibles en México.

La estrategia más viable de controlar a las royas es mediante el control genético, que se basa en incorporar genes efectivos de resistencia en el germoplasma de los diversos programas de mejoramiento genético de trigo que existen en México y el mundo. Para lo cual la búsqueda de germoplasma con resistencia a las razas más frecuente y con mayor espectro de virulencia es un 
trabajo constante. De las razas identificadas en la presente investigación la más frecuente fue la $\mathrm{CBJ} / \mathrm{QQ}$ y una de las más virulentas fue MCT/SP, para dichas razas se identificaron los genotipos con resistencia en plántula y planta adulta a roya de la hoja.

En el Cuadro 2 se indica el nivel de resistencia en plántula y planta adulta bajo condiciones de invernadero de ocho variedades de trigo harinero recomendadas para siembra bajo condiciones de riego en el Noroeste de México, de las cuales Conatrigo F2015 fue resistente en plántula y planta adulta a las dos razas, lo que indica que posee al menos un gen de resistencia efectivo a roya de la hoja.

Cuadro 2. Respuesta en plántula y planta adulta de genotipos de trigo harinero que pueden ser usados como progenitores, fuentes de resistencia y liberación de variedades.

\begin{tabular}{|c|c|c|c|c|c|}
\hline \multirow{2}{*}{ No. } & \multirow{2}{*}{ Variedad/genotipo } & \multicolumn{2}{|c|}{$\mathrm{MCT} / \mathrm{SP}$} & \multicolumn{2}{|c|}{$\mathrm{CBJ} / \mathrm{QQ}$} \\
\hline & & PLT & PA & PLT & $\mathrm{PA}$ \\
\hline 1 & Kronstad F2004 & 3 & $40 \mathrm{MS}$ & 1 & $10 \mathrm{MR}$ \\
\hline 2 & Roelfs F2007 & 3 & $50 \mathrm{~S}$ & 1 & $1 \mathrm{R}$ \\
\hline 3 & Villa Juárez F2009 & 3 & $20 \mathrm{MS}$ & $1-$ & $1 \mathrm{R}$ \\
\hline 4 & Borlaug 100 F2014 & 3 & $20 \mathrm{MS}$ & ; & $5 \mathrm{MR}$ \\
\hline 5 & Bacorehuis F2015 & $3+$ & 30MS & $1-$ & $5 \mathrm{MR}$ \\
\hline 6 & Conatrigo F2015 & $1-$ & $1 \mathrm{R}$ & $1-$ & $5 \mathrm{MR}$ \\
\hline 7 & Noreste F2018 & 3 & $40 \mathrm{MS}$ & $1-$ & $5 \mathrm{MS}$ \\
\hline 8 & Hans F2019 & $3+$ & $70 \mathrm{~S}$ & $1-$ & $5 \mathrm{MR}$ \\
\hline 9 & BECARD/FRNCLN//BORL14 & $X$ & $15 \mathrm{MR}$ & $1-$ & 0 \\
\hline 10 & CHEWINK \#1/MUTUS//MUTUS *2/HARIL\#1 & ; & 0 & 1 & $5 \mathrm{MR}$ \\
\hline 11 & MUTUS*2//ND643/2*WBLL1/3/BORL14 & ; & 0 & 0 & 0 \\
\hline 12 & FRET $2 * 2 / \mathrm{KIRITATI} / / \mathrm{KIRITATI} / 2 * \mathrm{TRCH} / 3 / \ldots$ & 3 & $30 \mathrm{MS}$ & $1-$ & $5 \mathrm{MR}$ \\
\hline 13 & NS.732/HER/3/PRL/SARA//TSI/VEE\#5/4/... & 3 & $30 \mathrm{MS}$ & $1-$ & $1 \mathrm{R}$ \\
\hline 14 & WBLL1*2/BRAMBLING//WBLL1 $* 2 / \ldots$ & $3+$ & $30 \mathrm{MS}$ & $1-$ & $5 \mathrm{MR}$ \\
\hline
\end{tabular}

$\mathrm{R}=$ resistente; $\mathrm{MR}=$ moderada resistencia.

Por otra parte, las variedades Villa Juárez F2009, Borlaug 100 y Bacorehuis F2015 fueron moderadamente susceptibles a la raza más virulenta (MCT/SP); sin embargo, con un comportamiento de moderada susceptibilidad a susceptible fueron las variedades Kronstad F2004, Roelfs F2007, Noreste F2018 y Hans F2019.

De las 27 líneas evaluadas solo en seis se observó reacción de resistencia, moderada resistencia y susceptibilidad. Las líneas 10 (CHEWINK \#1/MUTUS//MUTUS*2/HARIL\#1) y 11 (MUTUS*2//ND643/2*WBLL1/3/BORL14) fueron resistentes en plántula y planta adulta a las dos razas evaluadas lo que indica que poseen por lo menos un gen mayor efectivo de resistencia a roya de la hoja. 


\section{Conclusiones}

En el Valle del Carrizo y Valle del Fuerte durante el ciclo otoño-invierno, 2018-2019 se identificaron siete razas fisiológicas de roya de la hoja, de las cuales CBJ/QQ fue la más frecuente. CONATRIGO F2015 mostro resistencia en plántula y planta adulta a las razas CBJ/QQ y MCT/SP lo que indica que posee un gen mayor de resistencia a roya de la hoja. La mayoría de las razas identificadas presentaron un espectro de virulencia amplio. Las razas detectadas con baja frecuencia posiblemente no lleguen a ser dominantes en Sinaloa. Es de suma importancia identificar líneas con resistencia genética a las principales razas fisiológicas de royas que se presenten en el norte de Sinaloa, lo cual permitirá generar variedades que disminuyan el impacto económico causado por la roya de la hoja. La resistencia genética es una de las alternativas de manejo sustentable más viable para el control de las royas del trigo en México.

\section{Agradecimientos}

Al proyecto fiscal de trigo del INIFAP con No. SIGI: 22435234864.

\section{Literatura citada}

CESAVESIN. 2019. Comité Estatal de Sanidad Vegetal del Estado de Sinaloa. http://www.cesavesin.org.mx/cms/.

FAO. 2020. Food and Agriculture Organization of the United Nations. Crops. Rome, Italy. http://www.fao.org/faostat/en/\#data/QC.

Huerta-Espino, J. and Roelfs A. P. 1989. Physiological specialization on leaf rust on durum wheat. Phytopathology. 79(3):1218.

Huerta-Espino, J. and Singh, R. P. 1994. First report of virulence for wheat leaf rust resistance gene Lr19 in Mexico. Plant Disease. 7886):640-640. https://doi.org/10.1094/PD-78-0640C.

Huerta-Espino, J. y Singh P. R. 2000. Las royas del trigo. In: el trigo de temporal en México. Villaseñor M, Espitia, R. E. (Eds). SAGAR, INIFAP, CIR-CENTRO CEVAMEX. México, DF. 231-251 pp.

Huerta-Espino, J.; Singh, R. P.; Germán, S.; McCallum, B. D.; Park, R. F.; Chen, W. Q.; Bhardwaj, S. G. and Goyeau, H. 2011. Global status of wheat leaf rust caused by Puccinia triticina. Euphytica. 179(1):143-160. https://doi.org/10.1007/s10681-011-0361-X.

Huerta-Espino, J.; Singh, R. P.; Villaseñor, M. H. E.; Rodríguez, G. M. F.; Solís, M. E. y Espitia, R. E. 2020. Habilidad competitiva de razas de roya de la hoja provenientes de trigos cristalinos. Rev. Mex. Cienc. Agríc. 11(1):97-109. https://doi.org/10.29312/remexca.v11i1.1763.

Kolmer, J. A.; Long, D. L. and Hughes, M. E. 2011. Physiologic specialization of Puccinia triticina on wheat in the United States in 2009. Plant Dis. 95(8):935-940. https://doi.org/10.1094/PDIS-11-10-0786.

Kolmer, J. A. and Hughes, M. E. 2018. Physiologic specialization of Puccinia triticina on wheat in the United States in 2016. Plant Dis. 102(6):1066-1071. https://doi.org/10.1094/PDIS11-17-1701-SR.

Long, D. L. and Kolmer, J. A. 1989. A North American system of nomenclature for Puccinia recondita f. sp. tritici. Phytopathology. 79(5):525-529. 
McIntosh, R. A.; Dubcovsky, J.; Rogers, W. J.; Morris, C. and Xia, X. C. 2017. Catalogue of gene symbols for wheat. Supplement. 20 p.

Peterson, R. F.; Campbell, A. B. and Hannah, A. E. 1948. A diagrammatic scale for estimating rust intensity on leaves and stem of cereals. Canadian J. Res. 26c(5):496-500. https://doi.org/10.1139/cjr48c-033.

Rodríguez, G. M. F.; Huerta-Espino, J.; Villaseñor, M. H. E.; Sandoval, I. J. S. and Singh, R. 2010. Análisis de virulencia de la roya amarilla (Puccinia striiformis f. sp. tritici) del trigo (Triticum aestivum L.) en los Valles Altos de México. Agrociencia. 44(4):491-502.

Roelfs, A. P.; Singh, R. P. and Saari, E. E. 1992. Rust diseases of wheat: concepts and methods of disease management. CIMMYT. El Batán, Texcoco, Estado de México. Manual técnico 3. $81 \mathrm{p}$.

SIAP. 2020. Servicio de Información Agroalimentaria y Pesquera. Secretaría de Agricultura, Ganadería, Desarrollo Rural, Pesca y Alimentación (SAGARPA). México, DF. http://www.siap.sagarpa.gob.mx.

Singh, R. P. 1991. Pathogenicity variations of Puccinia recondita f. sp. tritici and P. graminis $\mathrm{f}$. sp. tritici in wheat-growing areas of Mexico during 1988 and 1989. Plant Dis. 75(8):790794. https://doi.org/10.1094/PD-75-0790.

Singh, R. P.; Huerta- Espino, J.; Bhavani, S.; Herrera-Foessel, S; Singh, A. D.; Singh, P. K.; Velu, G.; Mason, R. E; Jin, Y. and J. Crossa. 2011. Race non-specific resistance to rust diseases in CIMMYT spring wheats. Euphytica. 179(1):175-186. https://doi.org/10.1007/s10681010-0322-9.

Singh, R. P.; Singh, P. K.; Rutkoski, J.; Hodson, D. P.; Lee, X.; Jorgensen, L. N.; Hovmoller, M. S. and Huerta-Espino, J. 2016. Disease impact on wheat yield potential and prospects of genetic control. Annual Review of Phytopathology. 54(1):303-322. https://doi.org/10.1146/annurev-phyto-080615-095835.

Villaseñor-Espín O. M.; Huerta-Espino, J.; Leyva-Mir, S. G.; Villaseñor-Mir, H. E. y EspitiaRangel, E. 2003. Análisis de virulencia de la roya de la hoja (Puccinia triticina Eriks.) del trigo (Triticum aestivum L.) en los Valles Altos de México. Rev. Mex. Fitopatol. 21(1):56-62. 